\title{
A compendious summary of Parkinson's disease patient-derived iPSCs in the first decade
}

\author{
Chao Ren ${ }^{1,2,3 \#}$, Fen Wang ${ }^{3 \#}$, Li-Na Guan $^{1,4}$, Xiao-Yu Cheng $^{1}$, Cai-Yi Zhang ${ }^{5}$, De-Qin Geng ${ }^{6}$, \\ Chun-Feng Liu ${ }^{1,3}$
}

${ }^{1}$ Department of Neurology, The Second Affiliated Hospital of Soochow University, Suzhou 215004, China; ${ }^{2}$ Department of Neurology, The Affiliated Yantai Yuhuangding Hospital of Qingdao University, Yantai 264000, China; Jiangsu Key Laboratory of Neuropsychiatric Diseases and Institute of Neuroscience, Soochow University, Suzhou 215123, China; ${ }^{4}$ Department of Neurosurgical Intensive Care Unit, The Affiliated Yantai Yuhuangding Hospital of Qingdao University, Yantai 264000, China; ${ }^{5}$ Department of Emergency, ${ }^{6}$ Department of Neurology, The Affiliated Hospital of Xuzhou Medical University, Xuzhou 221006, China

Contributions: (I) Conception and design: CF Liu; (II) Administrative support: DQ Geng; (III) Provision of study materials or patients: LN Guan, XY Cheng, CY Zhang; (IV) Collection and assembly of data: C Ren, F Wang; (V) Data analysis and interpretation: C Ren, F Wang; (VI) Manuscript writing: All authors; (VII) Final approval of manuscript: All authors.

\#These authors contributed equally to this work.

Correspondence to: Chun-Feng Liu. Department of Neurology, The Second Affiliated Hospital of Soochow University, Suzhou 215004, China. Email: liuchunfeng@suda.edu.cn; De-Qin Geng. Department of Neurology, The Affiliated Hospital of Xuzhou Medical University, Xuzhou 221006, China. Email: gengdeqintg@126.com.

\begin{abstract}
The number of Parkinson's disease (PD) patients increases with aging, which brings heavy burden to families and society. The emergence of patient-derived induced pluripotent stem cells (iPSCs) has brought hope to the current situation of lacking new breakthroughs in diagnosis and treatment of PD. In this article, we reviewed and analyzed the current researches related to PD patient-derived iPSCs, in order to provide solid theoretical basis for future study of PD. In 2008, successful iPSCs derived from PD patients were reported. The current iPSCs research in PD mostly focused on the establishment of specific iPSCs models of PD patients carrying susceptible genes. The main source of PD patient-derived iPSCs is skin fibroblasts and the mainstream reprogramming methodology is the mature "four-factor" method, which introduces four totipotent correlation factors Oct4, Sox2, Klf4 and c-Myc into somatic cells. The main sources of iPSCs are patients with non-pedigrees and there have been no studies involving both PD patients and unaffected carriers within the same family. Most of the existing studies of PD patient-derived iPSCs started with the induction method for obtaining dopaminergic neurons in the first instance, but therapeutic applications are being increased. Although it is not the ultimate panacea, and there are still some unsolved problems (e.g., whether the mutated genes should be corrected or not), a better understanding of iPSCs may be a good gift for both PD patients and doctors due to their advantages in diagnosis and treatment of PD.
\end{abstract}

Keywords: Parkinson's disease (PD); induced pluripotent stem cells (iPSCs); differentiation; dopaminergic neurons (DANs); cell transplantation

Submitted Jul 09, 2019. Accepted for publication Oct 10, 2019.

doi: $10.21037 /$ atm.2019.11.16

View this article at: http://dx.doi.org/10.21037/atm.2019.11.16

\section{Introduction}

As one of the most common chronic progressive degenerative diseases of the nervous system in middle and old age, the incidence of Parkinson's disease (PD) is next only to Alzheimer's disease (AD). PD typically starts with neuromelanin-rich dopaminergic neurons (DANs) degeneration in the substantia nigra pars compacta of the midbrain and dopamine (DA) deficiency in the striatum (1), 
and when DANs in the substantia nigra are reduced by more than $50 \%$ and DA content is reduced by more than $70 \%, \mathrm{PD}$ patients will experience resting tremor, motor retardation, myotonia and abnormal posture and gait (2). Of course, it will also gradually spread throughout the brain causing a more generalized neuronal dysfunction and affecting other neurotransmitter systems, which is considered as the reason for most non-motor manifestations of the disease, such as depression, psychosis, cognitive decline, and dementia (3). The incidence of PD increases with age. Due to an increase in the aging population, the number of PD patients has increased year by year, and the long-term treatment and care of PD patients has led to a considerable economic and mental burden to families and society (4). Although the diagnosis of PD can be made according to characteristic clinical symptoms and the response to medications, at present, the treatment of PD mainly relies on levodopa-based drug therapy and deep brain stimulation-based surgical intervention, which only partially control clinical symptoms and do not achieve complete relief. In fact, the major problem in treating PD is the lack of disease-modifying therapies that would halt or decelerate progression. The average course of $\mathrm{PD}$ is only 6.9 years (5). According to the meta-analysis made by Pringsheim et al. (6) the survival rate of PD patients decreases by about $5 \%$ each year, and the mortality risk in younger patients is higher than that in elderly patients. Over the 200 years since the discovery of PD, scientists have made significant efforts to study the diagnosis and treatment of PD. Unfortunately, novel and significant breakthroughs are rarely reported. Therefore, further in-depth innovative research on the pathogenesis of PD is needed urgently in order to identify more effective intervention and prevention methods.

\section{Induced pluripotent stem cells (iPSCs) and PD}

It is known that research on central nervous system diseases is mainly carried out on three levels, individual level, tissue/ organ level and the cellular level. Firstly, the study of PD on the individual level mainly involves animal models such as the mouse model, which cannot fully reflect the phenotypic characteristics of human DANs. Secondly, tissue/organ level studies of PD are still mainly carried out via postmortem neuropathology. Although neuropathology plays an important or even critical role in the identification of diseases and their neurological impairment, it usually has poor predictive value and represents only the end of the disease or a certain disease stage. Thirdly, the brain cells required for cytological studies are difficult to obtain from living PD patients. Thus, all of these factors limit hinder the development progress of in PD-related research more or less. To address these limitations, the development of new methods for obtaining PD patients' own specific cells that can demonstrate the different stages of PD and that are available in large quantities, and the use of these cells to study PD-related mechanisms and identify new treatments are profoundly significant. The discovery of iPSCs and the development of iPSCs-related technologies have provided new insights $(7,8)$ into the research on PD as iPSCs can not only show disease-related pathophysiological changes of PD, but also served as a source of seed cells for cell transplantation in PD patients (Figure 1).

\section{Cellular reprogramming to acquire PD-specific iPSCs}

iPSCs are generated by introducing pluripotent genes such as Oct4, Sox 2, Klf4 and $c-M y c$ into mature somatic cells, so that they are reprogrammed and restored to the cell state with the characteristics of embryonic stem cells that can differentiate into multiple lineage cell types (9). In recent years, research into establishing a PD-specific iPSCs model by reprogramming PD patients' somatic cells have gradually increased. In 2008 (8) and 2009 (10), successful examples of iPSCs derived from PD patients were reported. Since then, successful acquisition of PD-specific iPSC has been increasingly reported, see Table 1 . Table 1 shows that the main source of PD patient-derived iPSCs is skin fibroblasts. The main reprogramming methodology is the mature "four-factor" method, which introduces the 4 totipotent correlation factors $O c t 4$, Sox $2, K l f 4$ and $c-M y c$ into somatic cells. With the identification and cloning of disease-related genes, the role of genetic factors in PD has attracted more and more attention. As shown in Table 1, the current hot topics in iPSCs research in PD mostly focused on the establishment of specific iPSCs models of PD patients carrying susceptible genes such as LRRK2, PARKIN, SNCA, $G B A, P I N K 1$ and others. Among them, the most frequently reported is PD-specific iPSCs from patients carrying gene mutations of LRRK2 (Figure 2). As is known, PD patients with gene mutations of $L R R K 2$ have the typical clinical manifestations of PD, which may be familial or sporadic, and have the age-dependent pathogenic characteristics. Thus, it may be an ideal model to study the interaction of multiple factors such as genetic, environmental and natural aging factors in PD in the future (77). We also found that the PD-specific iPSCs are mainly derived from 


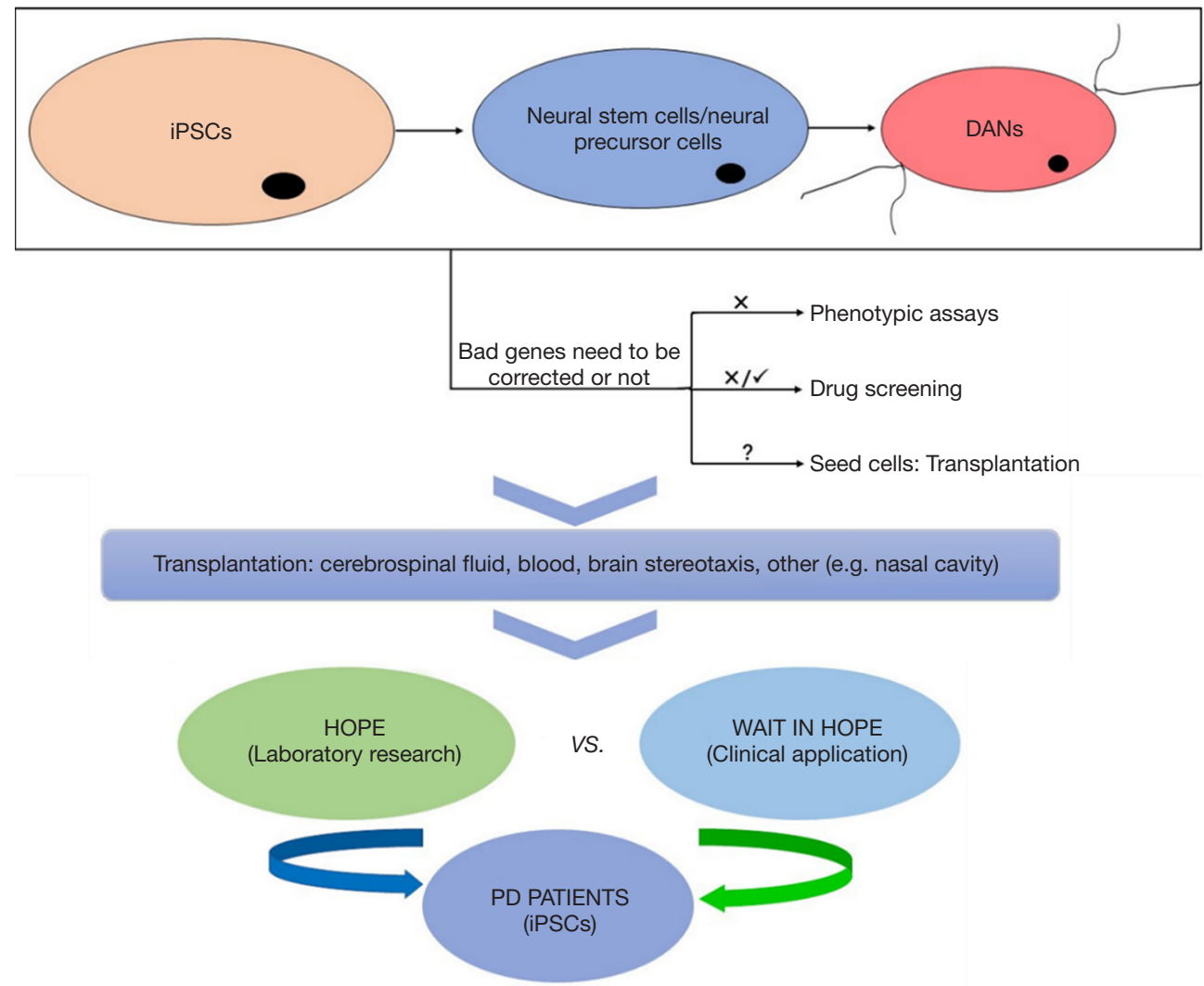

Figure 1 The use of iPSCs is bringing hope to PD patient. iPSC, induced pluripotent stem cell; PD, Parkinson's disease.

different pedigrees, but no studies have involved both PD patients and unaffected carriers within the same pedigree. Therefore, it will be of great importance to acquire a PD family with the same genetic mutant background and to use their iPSCs and related technologies to further study the pathogenesis of $\mathrm{PD}$, and then develop relevant prevention and control strategies, especially when there are both $\mathrm{PD}$ patients and unaffected carriers in the family.

\section{Showing disease-related pathophysiological changes of PD}

As a type of cells in vitro, iPSCs can not demonstrate the disease-related behaviors of PD like living animals, but it can show pathophysiological changes of PD $(28,78,79)$. Thus, more and more researchers $(67,80)$ believe that diseaserelated phenotypes analyses using $\mathrm{PD}$-specific iPSCs are useful in recapitulating the PD phenotypes (Table 2), which will help elucidate novel therapeutic targets. But sometimes, it should continue to be used in concert with other in vitro and animal models.

\section{Screening drugs for the treatment of PD}

Jang et al. (73) and Schüle et al. (81) suggested that diseasespecific iPSCs may be a platform for human disease modeling and drug discovery, but there are still a few limitations. The application of CRISPR/Cas9 (82) and a single cell high content assay (14) may provide new technologies to solve these limitations. In addition, other researchers (83) also believe that better regulation of the signal transduction pathways of FGF8, SHH, WNT and $\mathrm{BMP}$ is the key to ensure that iPSCs are used for drug screening. It can be concluded that the basis for the use of iPSCs in drug screening ultimately lies in the establishment of PD disease models (84). And most of cellular models of PD were established by PD patient-derived iPSCs with gene mutations (85). Furthermore, some drugs such as Coenzyme Q10, Rapamycin and GW5074 (a LRRK2 kinase inhibitor) have been screened using the related models (86).

Interestingly, Ryan et al. (87) found that MEF2C-PGC1 $\alpha$ pathway may be a novel therapeutic target to combat $\mathrm{PD}$ under gene-environmental interactions using small- 


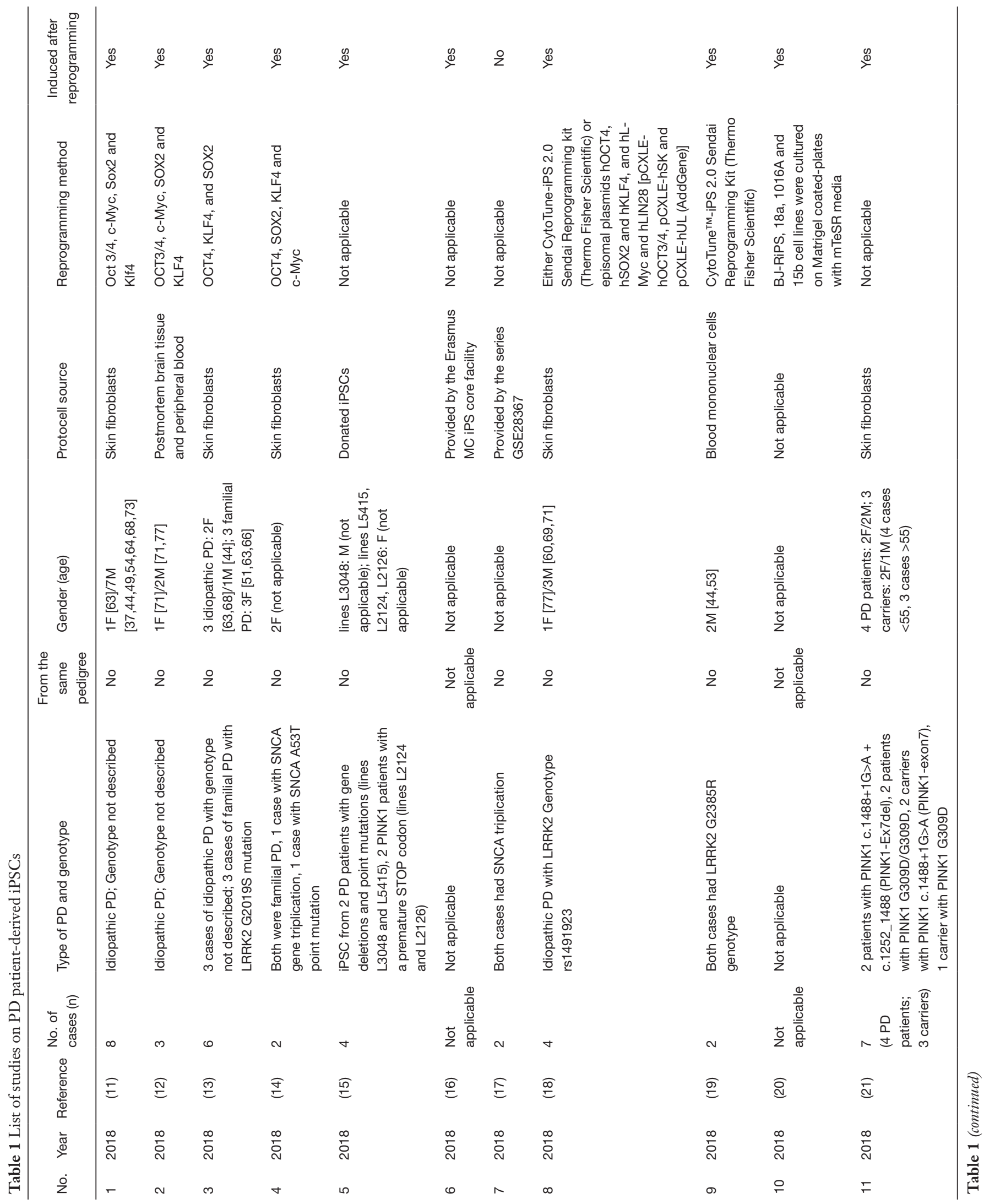




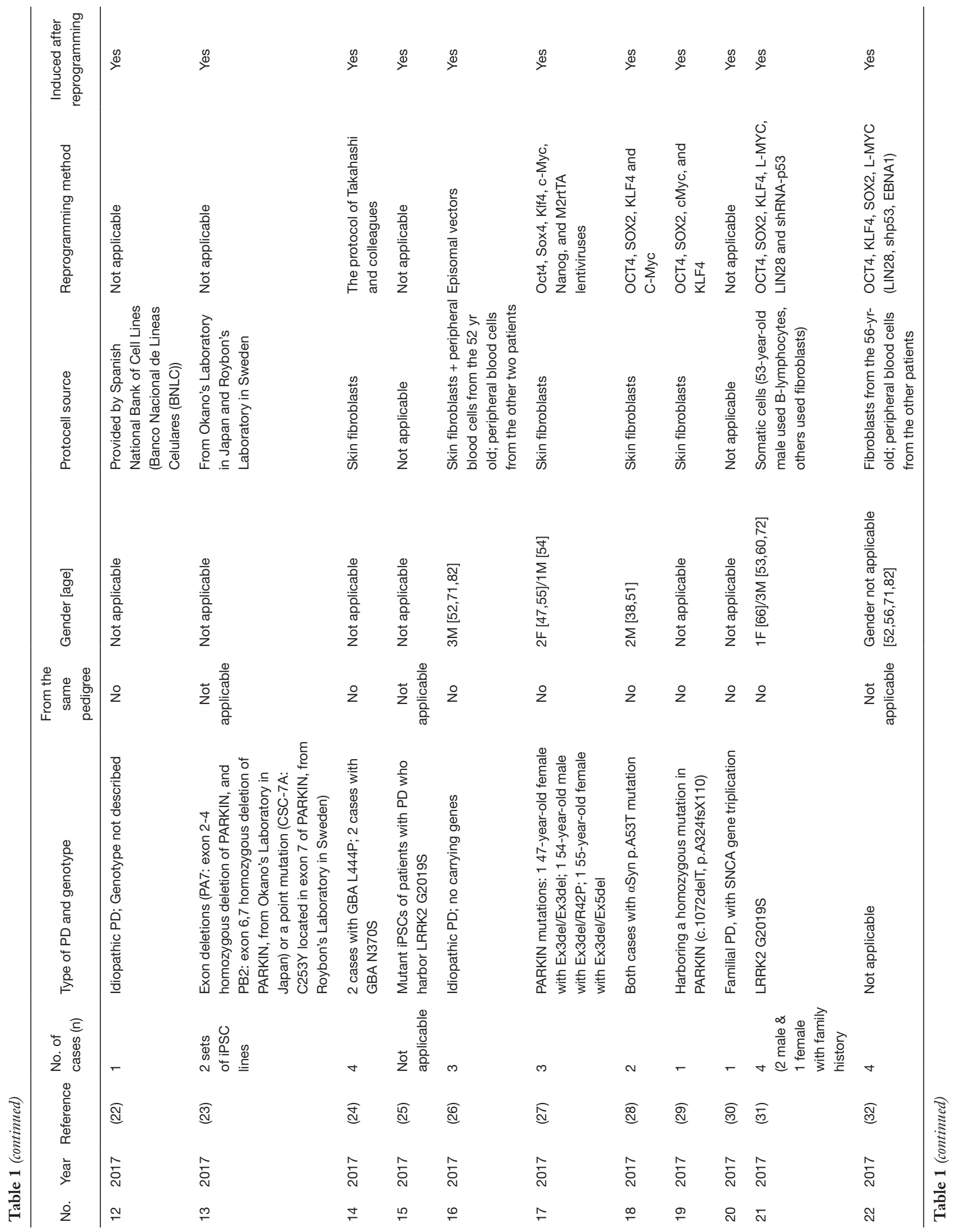




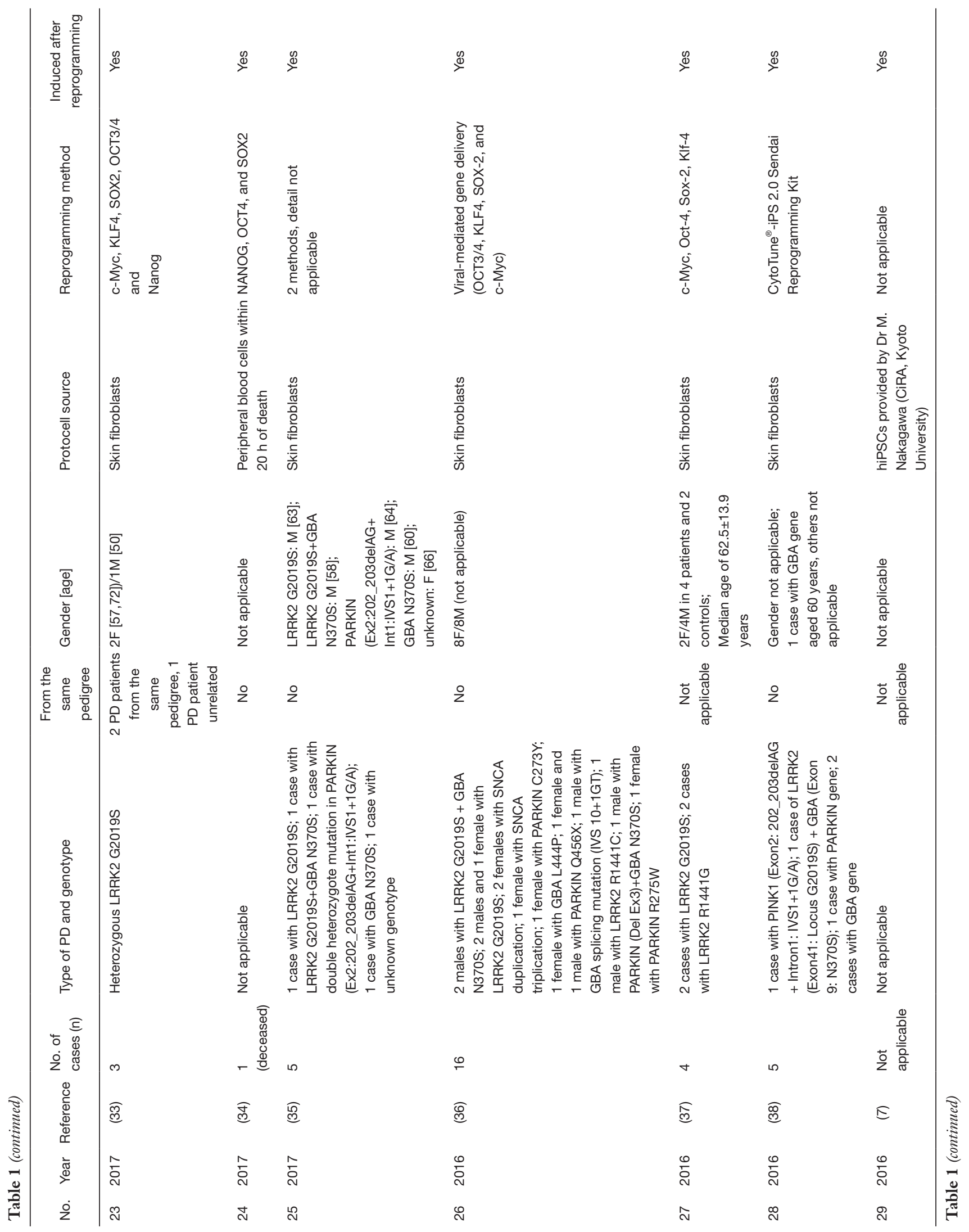




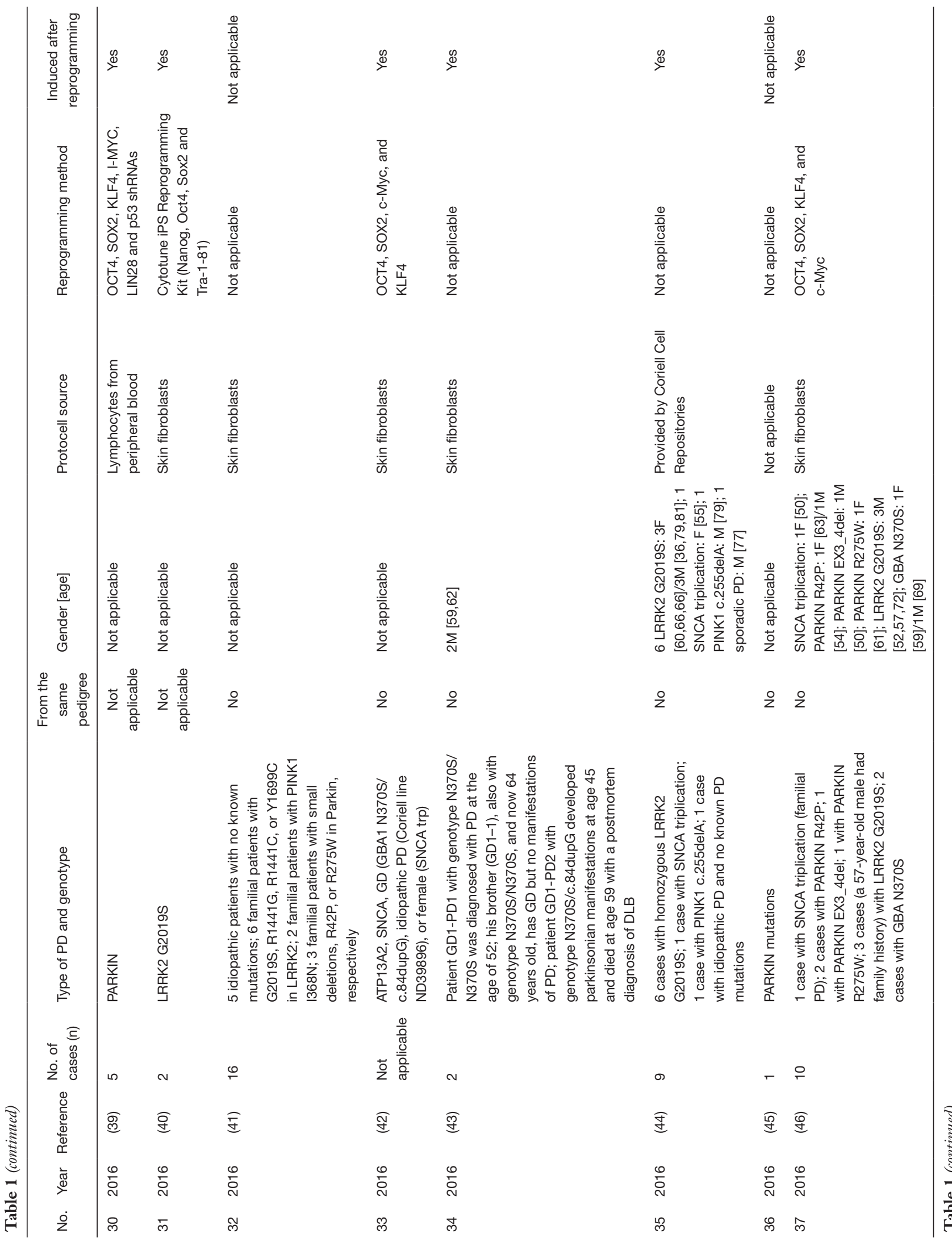




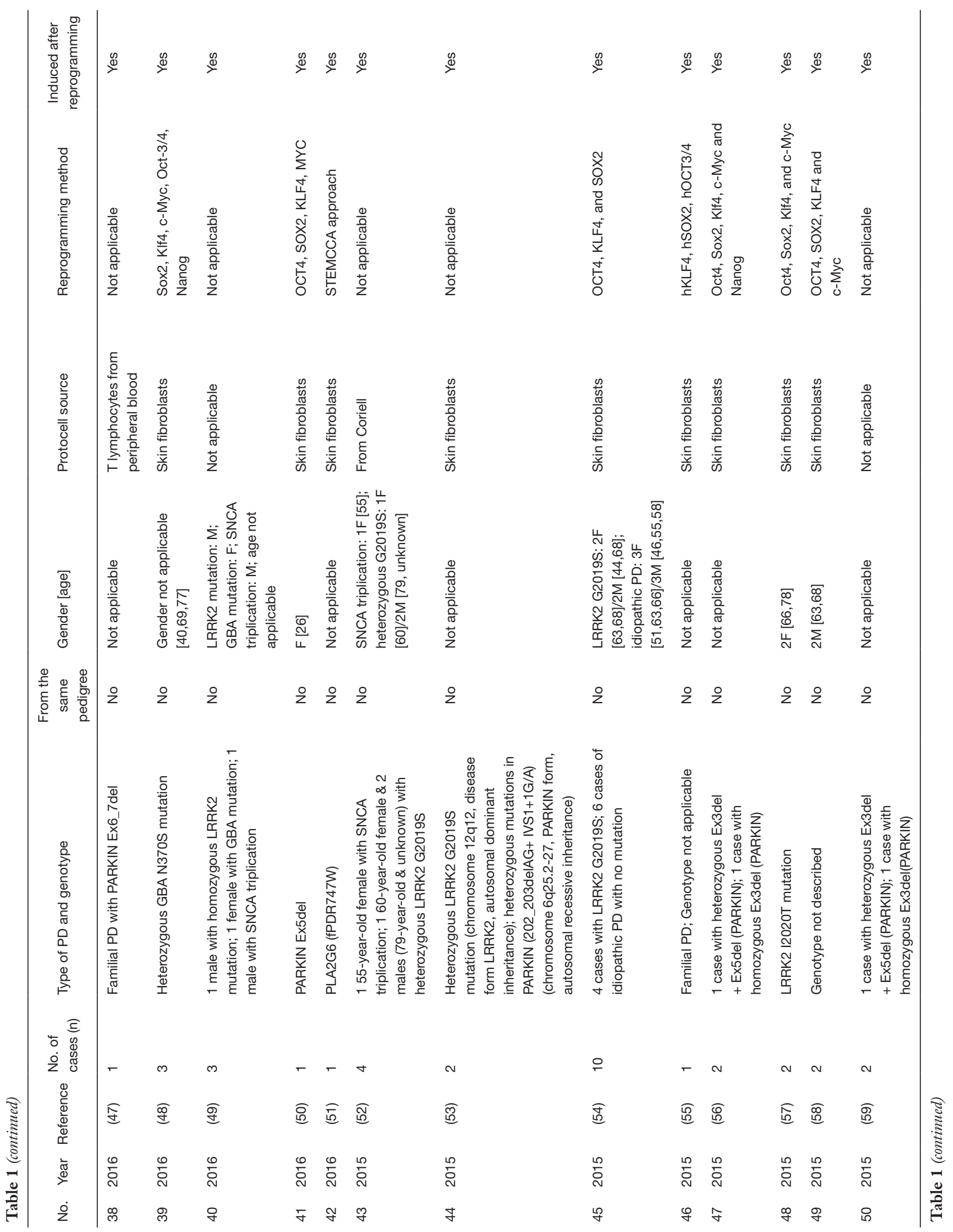









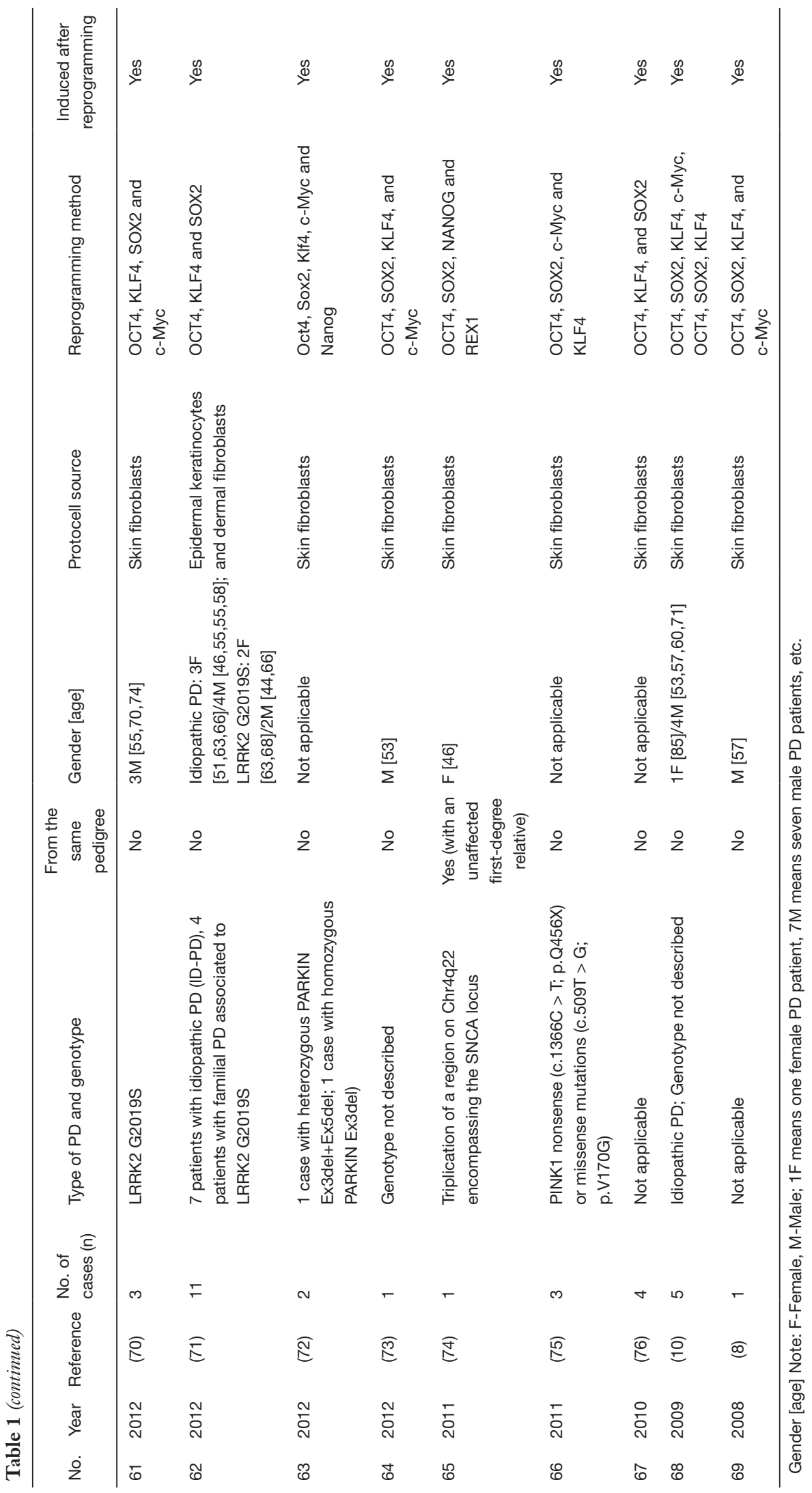




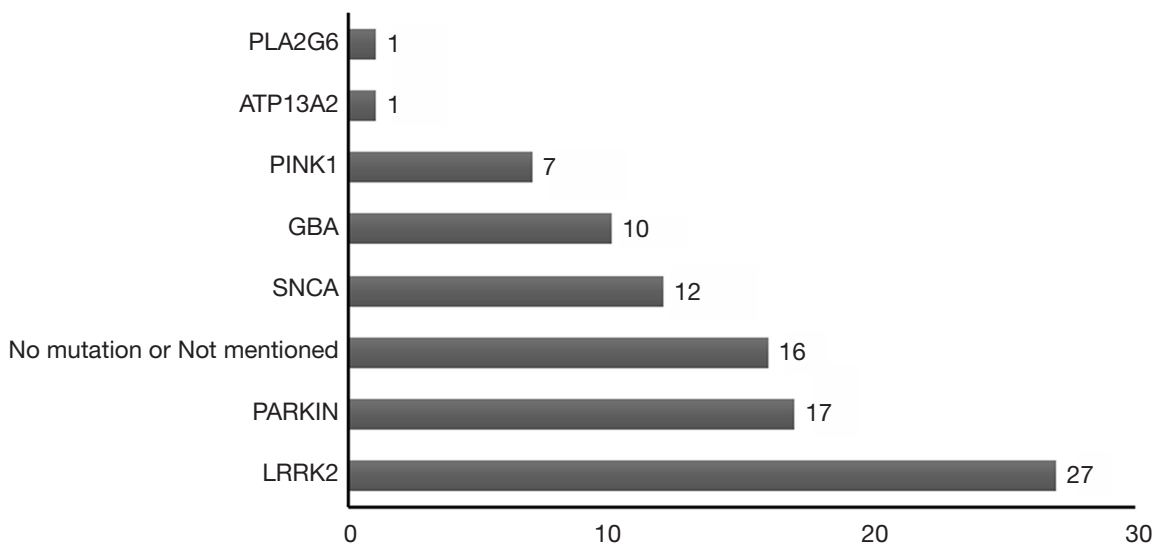

Figure 2 The number of studies reporting PD-specific iPSCs with different gene mutations. Note: one study may report two or more gene mutations of PD-specific iPSCs. iPSC, induced pluripotent stem cell; PD, Parkinson's disease.

Table 2 List of disease-related phenotypes reported in PD patient-derived iPSCs [modified and expanded after Jacobs BM (78)]

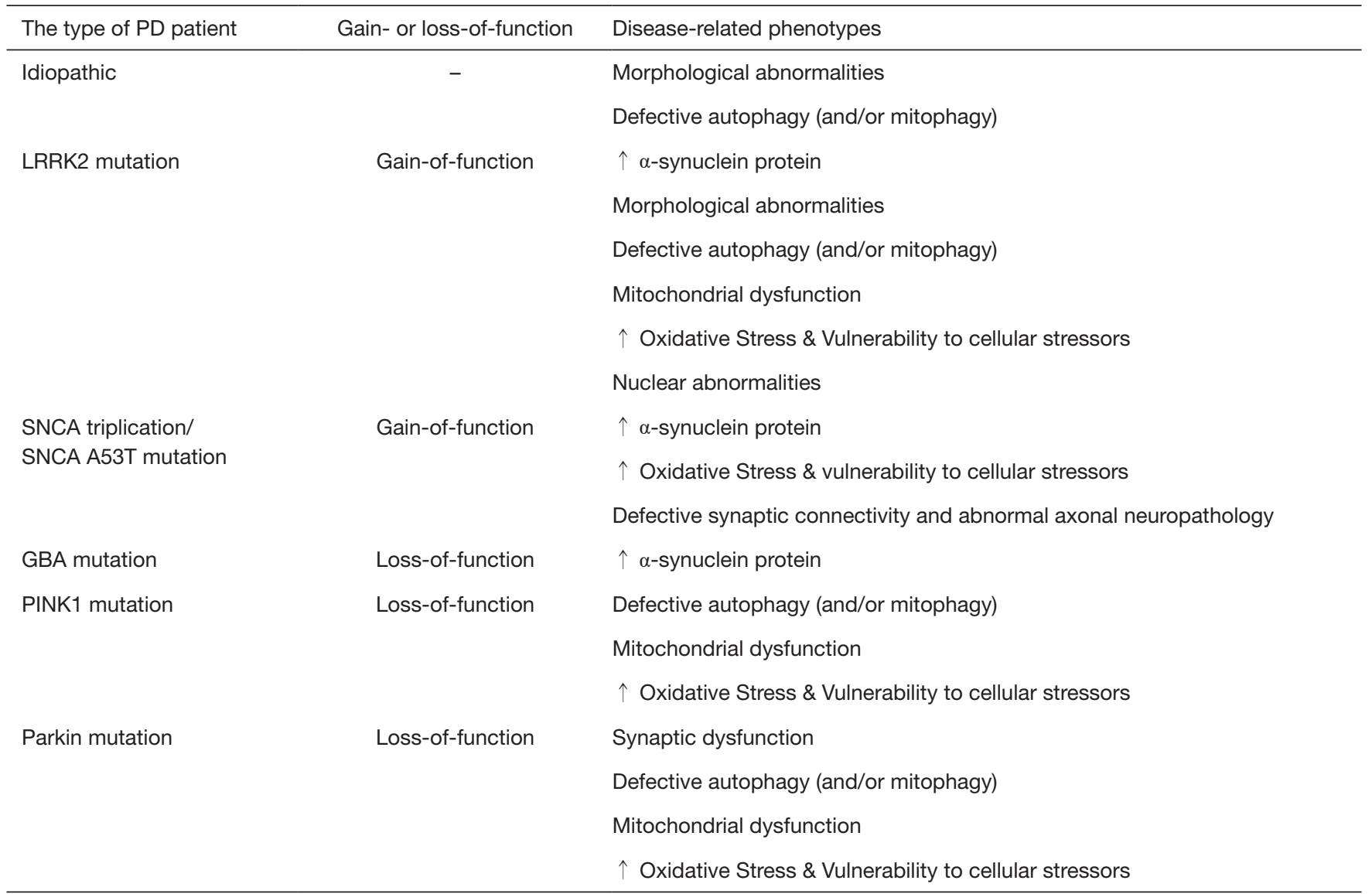


molecule high-throughput screening on a cellular model of PD established by PD patient-derived iPSCs.

\section{Using for cell transplantation in PD}

Most of the studies listed in Table 1 focused on the induction method for obtaining DANs at the beginning, but now the focus has been shifted to the application of PD patient-derived iPSCs application in the disease treatment. iPSCs overcome the lack of sources and ethical disputes of embryonic stem cells in terms of choosing seed cells for cell transplantation, as well as the difficulties in obtaining endogenous neural stem cells. Therefore, iPSCs are ideal seed cells for cell transplantation in PD patients (88). However, due to the introduction of exogenous genes and the instability of in vivo differentiation, iPSCs are not suitable for direct use in cell transplantation in patients with PD. Stable and efficient directional differentiation of iPSCs into DANs in vitro is a precondition and one of the most difficult problems and hot topics in cell transplantation for PD patients.

The efficient differentiation of pluripotent stem cells into DANs in vitro requires compliance with the physiological process of neural development. The nervous system of vertebrates consists of a variety of cell types that develop along the fixed position of the dorsal-ventral (D-V) axis and the anterior-posterior (A-P) axis of the neural canal. The mechanism for controlling this process is not fully understood. At present, it is believed that the signal center controlling the operation of these two main axes has established an epigenetic Cartesian coordinate "grid". As neural primordial cells have different positions in this grid, their different cell fates are determined. The epithelium, roof, floor plate and notochord of the dorsal ectoderm determine the fate of cells according to the $\mathrm{D}-\mathrm{V}$ axis. The paraxonic mesoderm of the prechordal plate, midbrain/ hindbrain junction (isthmus) and anterior nerve ridge (ANR) determine the fate of descendant cells along the A-P axis of the neural canal (89). In 2009, Chambers (90) transformed a high proportion of hES and hiPS into PAX6-positive A-P axonal precursor cells by adding two inhibitors of the SMAD signaling pathway (SB43542 and Noggin) in a monolayer adherent cell culture. The ratio of resultant DANs during the process of differentiation into lower-grade neurocytes from these cells was quite low. In 2011, Kriks et al. (91) added recombinant SHH and FGF8 into Chamber's induction protocol, and added the GSK/3 $\beta$ inhibitor CHIR99021 on day 3 of induction to activate the canonical Wnt signaling pathway, which efficiently induced the differentiation of pluripotent stem cells into FOXA2/LMX1A-positive floor plate-derived neural precursor cells, and induced their differentiation into a high proportion of TH-positive DANs. However, the underlying mechanism was not investigated. The canonical $\mathrm{Wnt} / \beta$-catenin signaling pathway plays an important role in biological development, cell transport, tumorigenesis and cell fate, as well as in roof plate and floor plate functions (92), and development of DANs in the central nervous system. In addition, Wnt can promote the neurogenesis of mesencephalic floor plate cells by antagonizing the SHH (93). Therefore, following the natural law of neural differentiation of human embryonic stem cells to induce PD patient-derived iPSCs to differentiate into DANs is the route that researchers must take. The protocol came from Nolbrant et al. (94) have suggested generation of precisely patterned neural cells from human pluripotent stem cells (hPSCs) is instrumental in developing disease models and stem cell therapies, but it must also follow the "law". Furthermore, DANs obtained by the above technique is a key step in establishing a PD disease model and in carrying out cell replacement therapy in the treatment of PD.

Encouragingly, a study on MPTP-PD monkey model of cell transplantation with human iPSCs-derived DANs carried out by Japanese scientists showed that the transplanted cells survived for at least two years and formed connections with the host monkey brains cells, but did not form any tumours. What is more, an increase in spontaneous movement of the monkeys after transplantation was witnessed (26). Immediately after the successful animal experiments the Japanese scientists started human research, and they implanted 'reprogrammed' stem cells into the brain of a patient with PD for the first time in 2018 October (as NEWS_Reported by Nature https://www. nature.com/articles/d41586-018-07407-9), which is the best gift for the Timeline: PD Patient-Derived iPSCs -The First Decade. But we can't be happy too soon. According to a recent study, control-derived grafts appeared to integrate better than PD [the p.A53T $\alpha$-synuclein $(\alpha$ Syn) mutation] grafts within the host tissue extending projections that formed more contacts with host striatal neurons (95), which could be ascribed to intrinsic properties of the iPSCslderived DANs that critically affected survival and proper neurite extension in the striatum after implantation (96). So, we always ought to keep calm down and ponder over each result of cell transplant with patient-derived iPSCs in PD! 


\section{Conclusions, problems and prospects}

PD patient-derived iPSCs have been studied for almost 10 years, and their reprogramming technology has become very mature. At present, in addition to the "fourfactor method" with routine use of skin fibroblasts, other programming methods are also gradually being optimized. The "only OCT4 factor" has already been used in the study of other iPSCs. The original source of mature somatic cells can also include blood, urine, teeth and other tissues. Furthermore, as mentioned above because of the exogenous gene introduction, more researchers have adopted alternative strategies to generate iPSCs, such as the nonintegration method $(97,98)$ and protein or peptide-based reprogramming (99). In conclusion, it is not difficult to obtain iPSCs from PD patients. However, it is still difficult to efficiently induce and obtain clinically available DANs for cell transplantation in PD. Furthermore, it is more difficult to make these DANs transplanted into PD patients reach the target site, achieve a long-term survival and play a therapeutic role (26).

In addition, although the disease model established by PD patient-derived iPSCs is an important and effective platform for studying the pathogenesis of $\mathrm{PD}$, for establishing a drug screening platform for PD treatment and for early diagnosis, it is not the ultimate panacea, and still has some limitations. Since PD-specific iPSCs could carry susceptible genes, is the mutation or deletion still detectable in the further induction? And whether or not gene correction required when abnormal gene mutation occurs? All of these need to be further investigated $(64,79)$.

Nevertheless, we should not ignore the critical studies (100-102) in which some authors think that organoid, especially brain organoid, may be better than the cultured cells for the treatment of nervous system diseases. Even as iPSCs-based models for neurodegenerative diseases, including $\mathrm{PD}$, have been repeatedly criticized because iPSCs-derived neurons are considered "young". Remarkably though, using such models a number of disease-associated phenotypes have been unraveled, suggesting that PD starts a lot earlier than initially thought, far earlier than the appearance of disease symptoms in patients and malfunctions can certainly be demonstrated in iPSCderived neurons. Not surprisingly, a recent report indicating aberrant mitochondrial morphology and functionality in iPSCs-derived neural precursors from PD patients (103). In summary, substantial efforts have been made in the application of PD patient-derived iPSCs (104). "Sometimes it might be better to leap before looking (105)", so it is most important to put the experimental results into clinical applications.

\section{Acknowledgments}

Funding: This work was supported by Suzhou Clinical Research Center of Neurological Disease (Award Number: Szzx2015033, recipient: Chun-Feng Liu), Jiangsu Provincial Medical Key Discipline Project (Award Number: ZDXKB2016022, recipient: Chun-Feng Liu) and Postgraduate Research \& Practice Innovation Program of Jiangsu Province (Award Number: KYCX19_1984, recipient: Chao Ren).

\section{Footnote}

Conflicts of Interest: The authors have no conflicts of interest to declare.

Ethical Statement: The authors are accountable for all aspects of the work in ensuring that questions related to the accuracy or integrity of any part of the work are appropriately investigated and resolved.

\section{References}

1. Giguère N, Burke Nanni S, Trudeau LE. On Cell Loss and Selective Vulnerability of Neuronal Populations in Parkinson's Disease. Front Neurol 2018;9:455.

2. Niu H, Shen L, Li T, et al. Alpha-synuclein overexpression in the olfactory bulb initiates prodromal symptoms and pathology of Parkinson's disease. Transl Neurodegener 2018;7:25.

3. Mao CJ, Chen JP, Zhang XY, et al. Parkinson's disease patients with pain suffer from more severe non-motor symptoms. Neurol Sci 2015;36:263-8.

4. Huse DM, Schulman K, Orsini L, et al. Burden of illness in Parkinson's disease. Mov Disord 2015;20:1449-54.

5. Pennington S, Snell K, Lee M, et al. The cause of death in idiopathic Parkinson's disease. Parkinsonism Relat Disord 2010;16:434-7.

6. Pringsheim T, Jette N, Frolkis A, et al. The prevalence of Parkinson's disease: a systematic review and meta-analysis. Mov Disord 2014;29:1583-90.

7. Samata B, Doi D, Nishimura K, et al. Purification of functional human ES and iPSC-derived midbrain dopaminergic progenitors using LRTM1. Nat Commun 
2016;7:13097.

8. Park IH, Arora N, Huo H, et al. Disease-Specific Induced Pluripotent Stem Cells. Cell 2008;134:877-86.

9. Papapetrou EP, Tomishima MJ, Chambers SM, et al. Stoichiometric and temporal requirements of Oct4, Sox2, Klf4, and c-Myc expression for efficient human iPSC induction and differentiation. PNAS 2009;106:12759-64.

10. Soldner F, Hockemeyer D, Beard C, et al. Parkinson's Disease Patient-Derived Induced Pluripotent Stem Cells Free of Viral Reprogramming Factors. Cell 2009;136:964-77.

11. Schulze M, Sommer A, Plötz S, et al. Sporadic Parkinson's disease derived neuronal cells show disease-specific mRNA and small RNA signatures with abundant deregulation of piRNAs. Acta Neuropathol Commun 2018;6:58.

12. Sommer A, Maxreiter F, Krach F, et al. Th17 Lymphocytes Induce Neuronal Cell Death in a Human iPSCBased Model of Parkinson's Disease. Cell Stem Cell 2018;23:123-31.e6.

13. Tolosa E, Botta-Orfila T, Morató X, et al. MicroRNA alterations in iPSC-derived dopaminergic neurons from Parkinson disease patients. Neurobiol Aging 2018;69:283-91.

14. Little D, Luft C, Mosaku O, et al. A single cell high content assay detects mitochondrial dysfunction in iPSCderived neurons with mutations in SNCA. Sci Rep 2018;8:9033.

15. Valadas JS, Esposito G, Vandekerkhove D, et al. ER Lipid Defects in Neuropeptidergic Neurons Impair Sleep Patterns in Parkinson's Disease. Neuron 2018;98:1155-69.e6.

16. Quadri M, Mandemakers W, Grochowska MM, et al. LRP10 genetic variants in familial Parkinson's disease and dementia with Lewy bodies: a genome-wide linkage and sequencing study. Lancet Neurol 2018;17:597-608.

17. George G, Singh S, Lokappa SB, et al. Gene co-expression network analysis for identifying genetic markers in Parkinson's disease - a three-way comparative approach. Genomics 2018. pii: S0888-7543(18)30282-9.

18. Marrone L, Bus C, Schöndorf D, et al. Generation of iPSCs carrying a common LRRK2 risk allele for in vitro modeling of idiopathic Parkinson's disease. Plos One 2018;13:e0192497.

19. Cheng YC, Huang CY, Ho MC, et al. Generation of 2 induced pluripotent stem cell lines derived from patients with Parkinson's disease carrying LRRK2 G2385R variant. Stem Cell Res 2018;28:1-5.

20. Paik EJ, O'Neil AL, Ng SY, et al. Using intracellular markers to identify a novel set of surface markers for live cell purification from a heterogeneous hIPSC culture. Sci Rep 2018;8:804.

21. Azkona G, López de Maturana R, Del Rio P, et al. LRRK2 Expression Is Deregulated in Fibroblasts and Neurons from Parkinson Patients with Mutations in PINK1. Mol Neurobiol 2018;55:506-16.

22. Costa-Besada MA, Valenzuela R, Garrido-Gil P, et al. Paracrine and Intracrine Angiotensin 1-7/Mas Receptor Axis in the Substantia Nigra of Rodents, Monkeys, and Humans. Mol Neurobiol 2018;5 5:5847-67.

23. Cartelli D, Amadeo A, Calogero AM, et al. Parkin absence accelerates microtubule aging in dopaminergic neurons. Neurobiol Aging 2018;61:66-74.

24. Straniero L, Rimoldi V, Samarani M, et al. The GBAP1 pseudogene acts as a ceRNA for the glucocerebrosidase gene GBA by sponging miR-22-3p. Sci Rep 2017;7:12702.

25. Yoon JH, Mo JS, Kim MY, et al. LRRK2 functions as a scaffolding kinase of ASK1-mediated neuronal cell death. Biochim Biophys Acta Mol Cell Res 2017;1864:2356-68.

26. Kikuchi T, Morizane A, Doi D, et al. Human iPS cellderived dopaminergic neurons function in a primate Parkinson's disease model. Nature 2017;548:592-6.

27. Zhong $\mathrm{P}, \mathrm{Hu} \mathrm{Z}$, Jiang $\mathrm{H}$, et al. Dopamine Induces Oscillatory Activities in Human Midbrain Neurons with Parkin Mutations. Cell Rep 2017;19:1033-44.

28. Kouroupi G, Taoufik E, Vlachos IS, et al. Defective synaptic connectivity and axonal neuropathology in a human iPSC-based model of familial Parkinson's disease. PNAS 2017;114:E3679-88.

29. Zanon A, Kalvakuri S, Rakovic A, et al. SLP-2 interacts with Parkin in mitochondria and prevents mitochondrial dysfunction in Parkin-deficient human iPSC-derived neurons and Drosophila. Hum Mol Genet 2017;26:2412-25.

30. Paillusson S, Gomez-Suaga P, Stoica R, et al. $\alpha$-Synuclein binds to the ER-mitochondria tethering protein VAPB to disrupt $\mathrm{Ca} 2+$ homeostasis and mitochondrial ATP production. Acta Neuropathol 2017;134:129-49.

31. Son MY, Sim H, Son YS, et al. Distinctive genomic signature of neural and intestinal organoids from familial Parkinson's disease patient-derived induced pluripotent stem cells. Neuropathol Appl Neurobiol 2017;43:584-603.

32. Kikuchi T, Morizane A, Doi D, et al. Idiopathic Parkinson's disease patient-derived induced pluripotent stem cells function as midbrain dopaminergic neurons in rodent brains. J Neurosci Res 2017;95:1829-37.

33. Sandor C, Robertson P, Lang C, et al. Transcriptomic profiling of purified patient-derived dopamine neurons 
identifies convergent perturbations and therapeutics for Parkinson's disease. Hum Mol Genet 2017;26:552-66.

34. Belle K, Shabazz FS, Nuytemans K, et al. Generation of disease-specific autopsy-confirmed iPSCs lines from postmortem isolated Peripheral Blood Mononuclear Cells. Neurosci Lett 2017;637:201-6.

35. Nenasheva VV, Novosadova EV, Makarova IV, et al. The Transcriptional Changes of trim Genes Associated with Parkinson's Disease on a Model of Human Induced Pluripotent Stem Cells. Mol Neurobiol 2017;54:7204-11.

36. Holmqvist $S$, Lehtonen $\breve{S}$, Chumarina $M$, et al. Creation of a library of induced pluripotent stem cells from Parkinsonian patients. NPJ Parkinsons Dis 2016;2:16009.

37. López de Maturana R, Lang V, Zubiarrain A, et al. Mutations in LRRK2 impair NF- $\kappa$ B pathway in iPSCderived neurons. J Neuroinflammation 2016;13:295.

38. Novosadova EV, Manuilova ES, Arsenyeva EL, et al. Fibroblast-like cells as an effective feeder for the cultivation and derivation of new lines of human induced pluripotent stem cells. Dokl Biochem Biophys 2016;470:353-6.

39. Fujimori K, Tezuka T, Ishiura H, et al. Modeling neurological diseases with induced pluripotent cells reprogrammed from immortalized lymphoblastoid cell lines. Mol Brain 2016;9:88.

40. Borgs L, Peyre E, Alix P, et al. Dopaminergic neurons differentiating from LRRK2 G2019S induced pluripotent stem cells show early neuritic branching defects. Sci Rep 2016;6:33377.

41. Hsieh CH, Shaltouki A, Gonzalez AE, et al. Functional Impairment in Miro Degradation and Mitophagy Is a Shared Feature in Familial and Sporadic Parkinson's Disease. Cell Stem Cell 2016;19:709-24.

42. Mazzulli JR, Zunke F, Tsunemi T, et al. Activation of $\beta$-Glucocerebrosidase Reduces Pathological $\alpha$-Synuclein and Restores Lysosomal Function in Parkinson's Patient Midbrain Neurons. J Neurosci 2016;36:7693-706.

43. Mazzulli JR, Zunke F, Tsunemi T, et al. Aflaki E, Borger DK, Moaven N, et al. A New Glucocerebrosidase Chaperone Reduces $\alpha$-Synuclein and Glycolipid Levels in iPSC-Derived Dopaminergic Neurons from Patients with Gaucher Disease and Parkinsonism. J Neurosci 2016;36:7441-52.

44. Lin L, Göke J, Cukuroglu E, et al. Molecular Features Underlying Neurodegeneration Identified through InVitro Modeling of Genetically Diverse Parkinson's Disease Patients. Cell Rep 2016;15:2411-26.

45. Gautier CA, Erpapazoglou Z, Mouton-Liger F, et al. The endoplasmic reticulum-mitochondria interface is perturbed in PARK2 knockout mice and patients with PARK2 mutations. Hum Mol Genet 2016;25:2972-84.

46. Momcilovic O, Sivapatham R, Oron TR, et al. Derivation, Characterization, and Neural Differentiation of Integration-Free Induced Pluripotent Stem Cell Lines from Parkinson's Disease Patients Carrying SNCA, LRRK2, PARK2, and GBA Mutations. Plos One 2016;11:e0154890.

47. Matsumoto T, Fujimori K, Andoh-Noda T, et al. Functional Neurons Generated from T Cell-Derived Induced Pluripotent Stem Cells for Neurological Disease Modeling. Stem Cell Reports 2016;6:422-35.

48. Fernandes HJ, Hartfield EM, Christian HC, et al. ER Stress and Autophagic Perturbations Lead to Elevated Extracellular $\alpha$-Synuclein inGBA-N370SParkinson's iPSC-Derived Dopamine Neurons. Stem Cell Reports 2016;6:342-56.

49. Xia N, Zhang P, Fang F, et al. Transcriptional comparison of human induced and primary midbrain dopaminergic neurons. Sci Rep 2016;6:20270.

50. Chang KH, Leechen GJ, Wu YR, et al. Impairment of proteasome and anti-oxidative pathways in the induced pluripotent stem cell model for sporadic Parkinson's disease. Parkinsonism Relat Disord 2016;24:81-8.

51. Zhou Q, Yen A, Rymarczyk G, et al. Impairment of PARK14-dependent $\mathrm{Ca}(2+)$ signalling is a novel determinant of Parkinson's disease. Nat Commun 2016;7:10332.

52. Schwab AJ, Ebert AD. Neurite Aggregation and Calcium Dysfunction in iPSC-Derived Sensory Neurons with Parkinson's Disease-Related LRRK2G2019S Mutation. Stem Cell Reports 2015;5:1039-52.

53. Konovalova EV, Novosadova EV, Grivennikov IA, et al. Phenotypical Differences in Neuronal Cultures Derived via Reprogramming the Fibroblasts from Patients Carrying Mutations in Parkinsonian Genes LRRK2, and PARK2. Bull Exp Biol Med 2015;159:772-5.

54. Fernández-Santiago R, Carballo-Carbajal I, Castellano G, et al. Aberrant epigenome in iPSC-derived dopaminergic neurons from Parkinson's disease patients. Embo Mol Med 2015;7:1529-46.

55. Djelloul M, Holmqvist S, Boza-Serrano A, et al. AlphaSynuclein Expression in the Oligodendrocyte Lineage: an In Vitro and In Vivo Study Using Rodent and Human Models. Stem Cell Reports 2015;5:174-84.

56. $\mathrm{Hu} \mathrm{Z}, \mathrm{Pu}$ J, Jiang H, et al. Generation of Naivetropic Induced Pluripotent Stem Cells from Parkinson's Disease Patients for High-Efficiency Genetic Manipulation and 
Disease Modeling. Stem Cells Dev 2015;24:2591-604.

57. Ohta E, Nihira T, Uchino A, et al. I2020T mutant LRRK2 iPSC-derived neurons in the Sagamihara family exhibit increased Tau phosphorylation through the AKT/GSK-3 $\beta$ signaling pathway. Hum Mol Genet 2015;24:4879-900.

58. Han F, Wang W, Chen B, et al. Human induced pluripotent stem cell-derived neurons improve motor asymmetry in a 6-hydroxydopamine-induced rat model of Parkinson's disease. Cytotherapy 2015;17:665-79.

59. Ren Y, Jiang H, Hu Z, et al. Parkin Mutations Reduce the Complexity of Neuronal Processes in iPSC-Derived Human Neurons. Stem Cells 2015;33:68-78.

60. Aboud AA, Tidball AM, Kumar KK, et al. PARK2 patient neuroprogenitors show increased mitochondrial sensitivity to copper. Neurobiol Dis 2015;73:204-12.

61. Schöndorf DC, Aureli M, McAllister FE, et al. iPSCderived neurons from GBA1-associated Parkinson's disease patients show autophagic defects and impaired calcium homeostasis. Nat Commun 2014;5:4028.

62. Chung CY, Khurana V, Auluck PK, et al. Identification and Rescue of $\alpha$-Synuclein Toxicity in Parkinson PatientDerived Neurons. Science 2013;342:983-7.

63. Sanders LH, Laganière J, Cooper O, et al. LRRK2 mutations cause mitochondrial DNA damage in iPSC-derived neural cells from Parkinson's disease patients: Reversal by gene correction. Neurobiol Dis 2014;62:381-6.

64. Reinhardt P, Schmid B, Burbulla LF, et al. Genetic correction of a LRRK2 mutation in human iPSCs links parkinsonian neurodegeneration to ERKdependent changes in gene expression. Cell Stem Cell 2013;12:354-67.

65. Orenstein SJ, Kuo SH, Tasset I, et al. Interplay of LRRK2 with chaperone-mediated autophagy. Nat Neurosci 2013;16:394-406.

66. Liu GH, Qu J, Suzuki K, et al. Progressive degeneration of human neural stem cells caused by pathogenic LRRK2. Nature 2012;491:603-7.

67. Imaizumi Y, Okada Y, Akamatsu W, et al. Mitochondrial dysfunction associated with increased oxidative stress and $\alpha$-synuclein accumulation in PARK2 iPSC-derived neurons and postmortem brain tissue. Mol Brain 2012;5:35.

68. Oliver Cooper, Hyemyung Seo, Shaida Andrabi, et al. Familial Parkinson's disease iPSCs show cellular deficits in mitochondrial responses that can be pharmacologically rescued. Sci Transl Med 2012;4:141ra90.

69. Luo Y, Fan Y, Chen X, et al. Generation of induced pluripotent stem cells from Asian patients with chronic neurodegenerative diseases. J Reprod Dev 2012;58:515-21.

70. Mak SK, Huang YA, Iranmanesh S, et al. Small Molecules Greatly Improve Conversion of Human-Induced Pluripotent Stem Cells to the Neuronal Lineage. Stem Cells Int 2012;2012:140427.

71. Sánchez-Danés A, Richaud-Patin Y, Carballo-Carbajal I, et al. Disease-specific phenotypes in dopamine neurons from human iPS-based models of genetic and sporadic Parkinson's disease. Embo Mol Med 2012;4:380-95.

72. Jiang H, Ren Y, Yuen EY, et al. Parkin Controls Dopamine Utilization in Human Midbrain Dopaminergic Neurons Derived from Induced Pluripotent Stem Cells. Nat Commun 2012;3:668.

73. Jang J, Yoo JE, Lee JA, et al. Disease-specific induced pluripotent stem cells: a platform for human disease modeling and drug discovery. Exp Mol Med 2012;44:202-13.

74. Devine MJ, Ryten M, Vodicka P, et al. Parkinson's disease induced pluripotent stem cells with triplication of the $\alpha$-synuclein locus. Nat Commun 2011;2:440.

75. Seibler P, Graziotto J, Jeong H, et al. Mitochondrial Parkin recruitment is impaired in neurons derived from mutant PINK1 iPS cells. J Neurosci 2011;31:5970-6.

76. Hargus G, Cooper O, Deleidi M, et al. Differentiated Parkinson patient-derived induced pluripotent stem cells grow in the adult rodent brain and reduce motor asymmetry in Parkinsonian rats. PNAS 2010;107:15921-6.

77. Ren C, Ding Y, Wei S, et al. G2019S Variation in LRRK2: An Ideal Model for the Study of Parkinson's Disease? Front Hum Neurosci 2019;13:306.

78. Jacobs BM. Stemming the hype: what can we learn from iPSC models of Parkinson's disease and how can we learn it? J Parkinsons Dis 2014;4:15-27.

79. Czaniecki C, Ryan T, Stykel MG, et al. Axonal pathology in hPSC-based models of Parkinson's disease results from loss of Nrf2 transcriptional activity at the Map1b gene locus. PNAS 2019;116:14280-9.

80. Nishimura K, Takahashi J. Therapeutic application of stem cell technology toward the treatment of Parkinson's disease. Biol Pharm Bull 2013;36:171-5.

81. Schüle B, Pera RA, Langston JW. Can cellular models revolutionize drug discovery in Parkinson's disease? Biochim Biophys Acta 2009;1792:1043-51.

82. Calatayud C, Carola G, Fernández-Carasa I, et al. CRISPR/Cas9-mediated generation of a tyrosine hydroxylase reporter iPSC line for live imaging and isolation of dopaminergic neurons. Sci Rep 2019;9:6811.

83. Brodski C, Blaess S, Partanen J, et al. Crosstalk of 
Intercellular Signaling Pathways in the Generation of Midbrain Dopaminergic Neurons In Vivo and from Stem Cells. J Dev Biol 2019. doi: 10.3390/jdb7010003.

84. Wang Y, Wang Z, Sun H, et al. Generation of induced pluripotent stem cell line (ZZUi007-A) from a 52-yearold patient with a novel CHCHD2 gene mutation in Parkinson's disease. Stem Cell Res 2018;32:87-90.

85. Ma D, Ng EY, Zeng L, et al. Development of a human induced pluripotent stem cell (iPSC) line from a Parkinson's disease patient carrying the N551K variant in LRRK2 gene. Stem Cell Res 2017;18:51-3.

86. Kang JF, Tang BS, Guo JF. The Progress of Induced Pluripotent Stem Cells as Models of Parkinson's Disease. Stem Cells Int 2016;2016:4126214.

87. Ryan SD, Dolatabadi N, Chan SF, et al. Isogenic human iPSC Parkinson's model shows nitrosative stressinduced dysfunction in MEF2-PGC1 $\alpha$ transcription. Cell 2013;155:1351-64.

88. Sundberg M, Bogetofte H, Lawson T, et al. Improved Cell Therapy Protocols for Parkinson's Disease Based on Differentiation Efficiency and Safety of hESC-, hiPSC-, and Non-Human Primate iPSC-Derived Dopaminergic Neurons. Stem Cells 2013;31:1548-62.

89. Ye W, Shimamura K, Rubenstein JL, et al. FGF and Shh Signals Control Dopaminergic and Serotonergic Cell Fate in the Anterior Neural Plate. Cell 1998;93:755-66.

90. Chambers SM, Fasano CA, Papapetrou EP, et al. Highly efficient neural conversion of human ES and iPS cells by dual inhibition of SMAD signaling. Nat Biotechnol 2009;27:275-80.

91. Kriks S, Shim JW, Piao J, et al. Dopamine neurons derived from human ES cells efficiently engraft in animal models of Parkinson's disease. Nature 2011;480:547-51.

92. Muroyama Y, Fujihara M, Ikeya M, et al. Wnt signaling plays an essential role in neuronal specification of the dorsal spinal cord. Genes Dev 2002;16:548-53.

93. Joksimovic M, Yun BA, Kittappa R, et al. Wnt antagonism of Shh facilitates midbrain floor plate neurogenesis. Nat Neurosci 2009;12:125-31.

94. Nolbrant S, Heuer A, Parmar M, et al. Generation of high-purity human ventral midbrain dopaminergic progenitors for in vitro maturation and intracerebral

Cite this article as: Ren C, Wang F, Guan LN, Cheng XY, Zhang CY, Geng DQ, Liu CF. A compendious summary of Parkinson's disease patient-derived iPSCs in the first decade. Ann Transl Med 2019;7(22):685. doi: 10.21037/atm.2019.11.16 transplantation. Nat Protoc 2017;12:1962-79.

95. Zygogianni O, Antoniou N, Kalomoiri M, et al. In Vivo Phenotyping of Familial Parkinson's Disease with Human Induced Pluripotent Stem Cells: A Proof-of-Concept Study. Neurochem Res 2019;44:1475-93.

96. Peng SP, Copray S. Comparison of Human Primary with Human iPS Cell-Derived Dopaminergic Neuron Grafts in the Rat Model for Parkinson's Disease. Stem Cell Rev Rep 2016;12:105-20.

97. Uhm KO, Jo EH, Go GY, et al. Generation of human induced pluripotent stem cells from urinary cells of a healthy donor using a non-integration system. Stem Cell Res 2017;21:44-6.

98. Scott E, Loya K, Mountford J, et al. MicroRNA regulation of endothelial homeostasis and commitment-implications for vascular regeneration strategies using stem cell therapies. Free Radic Biol Med 2013;64:52-60.

99. Liu H, Zeng F, Zhang M, et al. Emerging landscape of cell penetrating peptide in reprogramming and gene editing. $\mathrm{J}$ Control Release 2016;226:124-37.

100. Woodard CM, Campos BA, Kuo SH, et al. iPSCDerived Dopamine Neurons Reveal Differences between Monozygotic Twins Discordant for Parkinson's Disease. Cell Rep 2014;9:1173-82.

101. Nguyen HN, Byers B, Cord B, et al. LRRK2 Mutant iPSC-Derived DA Neurons Demonstrate Increased Susceptibility to Oxidative Stress. Cell Stem Cell 2011;8:267-80.

102. Steinbeck JA, Choi SJ, Mrejeru A, et al. Optogenetics enables functional analysis of human embryonic stem cell-derived grafts in a Parkinson's disease model. Nat Biotechnol 2015;33:204-9.

103. Walter J, Bolognin S, Antony PMA, et al. Neural Stem Cells of Parkinson's Disease Patients Exhibit Aberrant Mitochondrial Morphology and Functionality. Stem Cell Reports 2019;12:878-89.

104. Barker RA, Parmar M, Studer L, et al. Human Trials of Stem Cell-Derived Dopamine Neurons for Parkinson's Disease: Dawn of a New Era. Cell Stem Cell 2017;21:569-73.

105.Freedman MS, Uccelli A. Neurorepair with mesenchymal stem cells: hope or hype? Lancet Neurol 2012;11:123-5. 